\title{
Optimal controller gain tuning for robust stability of spacecraft formation
}

\author{
Esten I. Grøtli ${ }^{1}$, Antoine Chaillet ${ }^{2}$, Elena Panteley ${ }^{3}$, and J. Tommy Gravdahl ${ }^{1}$ \\ 1 Dept. of Eng. Cybernetics, O. S. Bragstads plass 2D, NTNU, 7491 Trondheim, NORWAY \\ 2 Univ. Paris Sud 11 - L2S - EECI - Supélec, 3 rue Joliot-Curie, 91192 GIF SUR YVETTE, \\ FRANCE \\ 3 CNRS - L2S, Supélec, 3 rue Joliot-Curie, 91192 GIF SUR YVETTE, FRANCE
}

\begin{abstract}
The spacecraft formation control problem sets high demands to the performance, especially with respect to positional accuracy. The problem is further complicated due to scarce fuel resources and limited actuation effects, in addition to the many sources of disturbances. This paper addresses the problem of finding the optimal gains of spacecraft formation controllers. By optimal, we mean the gains that minimizes a cost functional which penalizes both the control efforts and the state deviation, while still guaranteeing stability of the closed-loop systems in the presence of disturbances.
\end{abstract}

Keywords: Robustness, ISS, Moving average of disturbances, Spacecraft formation, gain tuning

\section{INTRODUCTION}

Formations of spacecraft are mainly motivated by their flexibility and increased baseline length compared to monolithic spacecraft. They are complex systems which require precise control to maintain relative trajectories, even in the presence of disturbances. These disturbances are often only described by the statistical or averaged characteristics of the perturbing signals (e.g. amplitude, energy, average energy, etc.), and are due to for instance intervehicle interference, solar wind and radiation and gravitational effects. To account for the limited fuel resources, the control law should minimize the fuel consumption, while still guaranteeing a predefined accuracy.

Ignoring the disturbances, an optimal control, that is the control input that minimizes some sensible cost function, can be derived using the Hamilton-Jacobi-Bellman equation or Pontryagin's maximum principle. With the introduction of $\mathcal{H}_{\infty}$ methods, by [19], one can specify the level of plant uncertainty and the signal gain from disturbance inputs to error outputs [4]. Although the $\mathcal{H}_{\infty}$ control problem was initially stated for systems described by transfer matrices, the ideas were soon translated into a state space setting [18], by realizing that the $\mathcal{H}_{\infty}$ norm is the $\mathcal{L}_{2}$ induced norm in the time domain. The nonlinear equivalent of the $\mathcal{H}_{\infty}$ problem was shown by [13] to be determined by the Hamilton-Jacobi-Isaacs (HJI) equation or inequality. An analytic solution to the HJI equation is general difficult to find. With the introduction of the inverse optimal control problem by [5], the performance index is a posteriori determined rather than a priori. 
In [10] this idea was applied to systems with disturbances, and it was shown that inputto-state stabilizability is a necessary and sufficient condition for what is known as the inverse optimal gain assignment problem to be solvable. The sufficiency part is relying on the Sontag type control law introduced in [16]. By solving the inverse optimal control problem solutions to a whole family of HJI equations are found. In [8] the link between optimality and closed-loop stability was further investigated by relating the cost function to a Lyapunov function, with the purpose of an optimal selection of the controller design parameters.

The problem of study in this paper is similar to the problem in [8], however we do not use the Lyapunov function as our cost function. Instead we introduce a new cost function, and solve the optimization problem with the stability constraints based on the Lyapunov analysis. The Lyapunov analysis of was performed in a previous paper by the authors, [6], and a guarantee for a hard bound on the state norm for input-to-state stable (ISS) systems in presence of signals with limited moving average were found. Furthermore, it was shown that the Lyapunov function can give an explicit estimate of the maximum of the disturbances' moving average that can be tolerated for a given precision. For the sake of completeness, some of the results are repeated here. In this paper we address the problem of finding the optimal gains, subject to constraints imposed by the Lyapunov analysis to achieve the above mentioned precision and stability. We consider a cost functional that is quadratic in both state and control variables, and the optimization is performed using fmincon, because of its ability to handle nonlinear constraints. We emphasize that we are not doing optimal control in usual sense, as the the controller and observer has already been designed without necessarily optimality in mind. It is obvious that the performance is restricted by parameterization of the chosen control law.

Notation and terminology A continuous function $\alpha: \mathbb{R}_{\geq} \rightarrow \mathbb{R}_{\geq 0}$ is of class $\mathcal{K}(\alpha \in$ $\mathcal{K}$ ), if it is strictly increasing and $\alpha(0)=0$. If, in addition, $\alpha(s) \rightarrow \infty$ as $s \rightarrow \infty$, then $\alpha$ is of class $\mathscr{K}_{\infty}\left(\alpha \in \mathscr{K}_{\infty}\right)$. A continuous function $\beta: \mathbb{R}_{\geq 0} \times \mathbb{R}_{\geq 0} \rightarrow \mathbb{R}_{\geq 0}$ is said to be of class $\mathcal{K} \mathcal{L}$ if, $\beta(\cdot, t) \in \mathcal{K}$ for any $t \in \mathbb{R}_{\geq 0}$, and $\beta(s, \cdot)$ is decreasing and tends to zero as $s$ tends to infinity. The solutions of the differential equation $\dot{x}=f(x, u)$ with initial condition $x_{0} \in \mathbb{R}^{n}$ is denoted by $x\left(\cdot ; x_{0}, u\right)$. We use $|\cdot|$ for the Euclidean norm of vectors and the induced norm of matrices. The closed ball in $\mathbb{R}^{n}$ of radius $\delta \geq 0$ centered at the origin is denoted by $\mathcal{B}_{\delta}$, i.e. $\mathcal{B}_{\delta}:=\left\{x \in \mathbb{R}^{n}:|x| \leq \delta\right\} .|\cdot|_{\delta}$ denotes the distance to the ball $\mathcal{B}_{\delta}$, that is $|x|_{\delta}:=\inf _{z \in \mathcal{B}_{\delta}}|x-z|$. $\mathcal{U}$ denotes the set of all measurable locally essentially bounded signals $u: \mathbb{R}_{\geq 0} \rightarrow \mathbb{R}^{p}$. For a signal $u \in \mathcal{U},\|u\|_{\infty}:=\operatorname{ess} \sup _{t \geq 0}|u(t)|$. The maximum and minimum eigenvalues of a symmetric matrix $A$ are denoted by $\lambda_{\max }(A)$ and $\lambda_{\min }(A)$, respectively. $I_{n}$ and $0_{n}$ denote the identity and zero matrices of $\mathbb{R}^{n \times n}$ respectively. We use $\mathrm{E}$ for the expectancy operator.

\section{ISS SYSTEMS AND SIGNALS WITH LOW MOVING AVERAGE}

We start by recalling some classical definitions related to the stability and robustness of nonlinear systems of the form

$$
\dot{x}=f(x, u),
$$


where $x \in \mathbb{R}^{n}, u \in \mathcal{U}$ and $f: \mathbb{R}^{n} \times \mathbb{R}^{p} \rightarrow \mathbb{R}^{n}$ is locally Lipschitz and satisfies $f(0,0)=0$.

Definition 1. Let $\delta$ be a nonnegative constant and $\mathcal{W} \subset \mathcal{U}$. The ball $\mathcal{B}_{\delta}$ is said to be globally exponentially stable (GES) for (1) with respect to $\mathcal{W}$ if there exists some positive constants $k_{1}$ and $k_{2}$ such that the solution of (1) satisfies

$$
\left|x\left(t ; x_{0}, u\right)\right| \leq \delta+k_{1}\left|x_{0}\right| e^{-k_{2} t}, \quad \forall t \geq 0 .
$$

for all $x_{0} \in \mathbb{R}^{n}$ and all $u \in \mathcal{W}$.

We next recall the definition of ISS, originally introduced in [15].

Definition 2. The system $\dot{x}=f(x, u)$ is said to be input-to-state stable (ISS) if there exist $\beta \in \mathcal{K} \mathcal{L}$ and $\gamma \in \mathcal{K}_{\infty}$ such that, for all $x_{0} \in \mathbb{R}^{n}$ and all $u \in \mathcal{U}$, the solution of (1) satisfies

$$
\left|x\left(t ; x_{0}, u\right)\right| \leq \beta\left(\left|x_{0}\right|, t\right)+\gamma\left(\|u\|_{\infty}\right), \quad \forall t \geq 0 .
$$

ISS thus imposes an asymptotic decay of the norm of the state up to a function of the amplitude $\|u\|_{\infty}$ of the input signal.

We also recall the following well-known Lyapunov characterization of ISS, originally established in [12] and thus extending the original characterization proposed by Sontag in [17].

Proposition 1. The system (1) is ISS if and only if there exist $\underline{\alpha}, \bar{\alpha}, \gamma \in \mathcal{K}_{\infty}$ and $\kappa>0$ such that, for all $x \in \mathbb{R}^{n}$ and all $u \in \mathbb{R}^{p}$,

$$
\begin{gathered}
\underline{\alpha}(|x|) \leq V(x) \leq \bar{\alpha}(|x|) \\
\frac{\partial V}{\partial x}(x) f(x, u) \leq-\kappa V(x)+\gamma(|u|) .
\end{gathered}
$$

$\gamma$ is then called a supply rate for (1).

The input signals we consider in this paper are slightly more restrictive than those in for instance [1], but the advantage is that a hard bound on the state norm can be guaranteed. Namely, we consider input signals with bounded moving average.

Definition 3. Given some constants $E, T>0$ and some function $\gamma \in \mathcal{K}$, the set $\mathcal{W}_{\gamma}(E, T)$ denotes the set of all signals $u \in \mathcal{U}$ satisfying

$$
\int_{t}^{t+T} \gamma(|u(s)|) d s \leq E, \quad \forall t \in \mathbb{R}_{\geq 0} .
$$

The main concern here is the measure $E$ of the maximum energy that can be fed into the system over a moving time window of given length $T$. These quantities are the only information on the disturbances that are taken into account in the control design. More parsimonious control laws than those based on the disturbances' amplitude or energy can therefore be expected. We stress that signals of this class are not necessarily globally essentially bounded, nor are they required to have a finite energy. Robustness to this class of signals thus constitutes an extension of the typical properties of ISS systems.

With input signals with bounded moving average, the following result of [6] guarantees global exponential stability of some neighborhood of the origin. 
Corollary 1. Assume there exists a continuously differentiable function $V: \mathbb{R}^{n} \rightarrow \mathbb{R}_{\geq 0}$, class $\mathcal{K}_{\infty}$ function $\gamma$, functions $\underline{\alpha}(s)=\underline{c} s^{p}$ and $\bar{\alpha}(s)=\bar{c} s^{p}$, with $\underline{c}, \bar{c}, p$ positive constants, and a positive constant $\kappa$ such that (4) and (5) hold for all $x \in \mathbb{R}^{n}$ and all $u \in \mathbb{R}^{p}$. Then, given any $T, \delta>0$, the ball $\mathcal{B}_{\delta}$ is $G E S$ for (1) with any signal $u \in \mathcal{W}_{\gamma}(E, T)$ provided that

$$
E(T, \delta) \leq \frac{c}{2} \delta^{p} \frac{e^{\kappa T}-1}{2 e^{\kappa T}-1}
$$

\section{SPACECRAFT FORMATION CONTROL}

The results of Section 2 were in [6] exploited to demonstrate robustness of a spacecraft formation control in a leader-follower configuration. We will here only include the parts necessary to keep this section self-contained. For further details, the reader is referred to the original paper.

\subsection{Spacecraft models}

The model for the leader spacecrafts motion with respect to a moving coordinate frame is given by

$$
\ddot{p}+C\left(\dot{\mathrm{v}}_{o}\right) \dot{p}+D\left(\dot{\mathrm{v}}_{o}, \ddot{\mathrm{v}}_{o}\right) p+n\left(r_{o}, p\right)=F_{l}
$$

while the model for follower spacecraft with respect to the leader is given by

$$
\ddot{\rho}+C\left(\dot{\mathrm{v}}_{o}\right) \dot{\rho}+D\left(\dot{\mathrm{v}}_{o}, \ddot{\mathrm{v}}_{o}\right) \rho+n\left(r_{o}+p, \rho\right)=F_{f}-F_{l},
$$

where $F_{l}:=\left(u_{l}+d_{l}\right) / m_{l}, F_{f}:=\left(u_{f}+d_{f}\right) / m_{f}$, and where subscripts $l$ and $f$ stand for the leader and follower spacecraft respectively. $p$ is the position of the leader spacecraft with respect to a coordinate frame in a Keplerian orbit, where as $\rho$ is the position of the follower spacecraft with respect to the leader. $m_{l}$ and $m_{f}$ are the spacecraft' masses, $u_{l}$ and $u_{f}$ are the control inputs, and $d_{l}$ and $d_{f}$ denote all exogenous perturbations acting on the spacecraft. Furthermore,

$$
\begin{gathered}
C\left(\dot{\mathrm{v}}_{o}\right):=2 \dot{\mathrm{v}}_{o} \bar{C}, \quad \bar{C}:=\left[\begin{array}{ccc}
0 & -1 & 0 \\
1 & 0 & 0 \\
0 & 0 & 0
\end{array}\right], \\
D\left(\dot{\mathrm{v}}_{o}, \ddot{\mathrm{v}}_{o}\right):=\dot{\mathrm{v}}_{o}^{2} \bar{D}+\ddot{\mathrm{v}}_{o} \bar{C}, \quad \bar{D}:=\operatorname{diag}(-1,-1,0),
\end{gathered}
$$

and

$$
n\left(r_{o}, p\right):=\mu\left(\frac{r_{o}+p}{\left|r_{o}+p\right|^{3}}-\frac{r_{o}}{\left|r_{o}\right|^{3}}\right),
$$

with $r_{o}$ being the origin of the coordinate reference frame, and $v_{o}$ the true anomaly. We make the following assumptions on the first and second derivatives of $\mathrm{v}_{o}$ :

Assumption 1 The true anomaly rate $\dot{\mathrm{v}}_{o}$ and true anomaly rate-of-change $\ddot{\mathrm{v}}_{o}$ of the reference frame satisfy $\left\|\dot{\mathrm{v}}_{o}\right\|_{\infty} \leq \beta_{\dot{v}_{o}}$ and $\left\|\ddot{\mathrm{v}}_{o}\right\|_{\infty} \leq \beta_{\ddot{v}_{o}}$, for some positive constants $\beta_{\dot{v}_{o}}$ and $\beta_{\ddot{v}_{o}}$. 


\subsection{Controller and observer design}

In [6] the proposed controller of the leader spacecraft were:

$$
u_{l}=m_{l}\left[\ddot{p}_{d}+C\left(\dot{\mathrm{v}}_{o}\right) \dot{p}_{d}+D\left(\dot{\mathrm{v}}_{o}, \ddot{\mathrm{v}}_{o}\right) p+n\left(r_{o}, p\right)-k_{l}\left(\dot{p}_{0}-\dot{p}_{r}\right)\right]
$$

with $p_{d}$ being the reference trajectory, $e_{l}=p-p_{d}$ the tracking error, $\tilde{p}=p-\hat{p}$ the estimation error, $\dot{p}_{r}=\dot{p}_{d}-\ell_{l} e_{l}, \dot{p}_{0}=\dot{\hat{p}}-\ell_{l} \tilde{p}$, and with velocity estimate provided by

$$
\begin{aligned}
\dot{\hat{p}} & =a_{l}+\left(l_{l}+\ell_{l}\right) \tilde{p} \\
\dot{a}_{l} & =\ddot{p}_{d}+l_{l} \ell_{l} \tilde{p} .
\end{aligned}
$$

The controller for the follower spacecraft has a similar structure, given by

$$
\begin{aligned}
u_{f}= & m_{f}\left[\ddot{p}_{d}+\ddot{\rho}_{d}+C\left(\dot{\mathrm{v}}_{o}\right)\left(\dot{p}_{d}+\dot{\rho}_{d}\right)+D\left(\dot{\mathrm{v}}_{o}, \ddot{\mathrm{v}}_{o}\right)(p+\rho)+n\left(r_{o}+p, \rho\right)+n\left(r_{o}, p\right)\right. \\
& \left.-k_{l}\left(\dot{p}_{0}-\dot{p}_{r}\right)-k_{f}\left(\dot{\rho}_{0}-\dot{\rho}_{r}\right)\right]
\end{aligned}
$$

with $\rho$ being the reference trajectory, $e_{f}=\rho-\rho_{d}$ the follower spacecraft tracking error, $\tilde{\rho}=\rho-\hat{\rho}$ the estimation error, auxiliary signals $\dot{\rho}_{r}=\dot{\rho}_{d}-\ell_{f} e_{f}$ and $\dot{\rho}_{0}=\dot{\hat{\rho}}-\ell_{f} \tilde{\rho}$, and with the observer

$$
\begin{aligned}
\dot{\hat{\rho}} & =a_{f}+\left(l_{f}+\ell_{f}\right) \tilde{\rho} \\
\dot{a}_{f} & =\ddot{\rho}_{d}+l_{f} \ell_{f} \tilde{\rho}
\end{aligned}
$$

The parameters $k_{l}, l_{l}, \ell_{l}, k_{f}, l_{f}$ and $\ell_{f}$ denote some positive tuning gains. The closedloop system may now be summarized into the equations

$$
\dot{X}=A\left(\dot{\mathrm{v}}_{o}(t), \theta\right) X+B d,
$$

where $X:=\left(X_{l}^{\top}, X_{f}^{\top}\right)^{\top}$ with $X_{l}:=\left(e_{l}^{\top}, \dot{e}_{l}^{\top}, \tilde{p}^{\top}, \dot{\tilde{p}}^{\top}\right)^{\top}$ and $X_{f}:=\left(e_{f}^{\top}, \dot{e}_{f}^{\top}, \tilde{\rho}^{\top}, \dot{\tilde{\rho}}^{\top}\right)^{\top}, d:=$ $\left(d_{l}^{\top}, d_{f}^{\top}\right)^{\top}, \theta:=\left(k_{l}, \ell_{l}, l_{l}, k_{f}, \ell_{f}, l_{f}\right)^{\top}$ and $A:=\operatorname{blckdiag}\left(A_{l}, A_{f}\right)$ with

$$
A_{i}\left(\dot{\mathrm{v}}_{o}\right):=\left[\begin{array}{cccc}
0_{3} & I_{3} & 0_{3} & 0_{3} \\
a_{21} & a_{22}\left(\dot{\mathrm{v}}_{o}\right) & a_{23} & a_{24} \\
0_{3} & 0_{3} & 0_{3} & I_{3} \\
a_{41} & a_{42}\left(\dot{\mathrm{v}}_{o}\right) & a_{43} & a_{44}
\end{array}\right]
$$

for $i \in\{l, f\}$. Out of notational compactness, the following matrices have been used: $a_{21}:=a_{41}:=-k_{i} \ell_{i} I_{3}, a_{22}:=a_{42}:=-C\left(\dot{\mathrm{v}}_{o}\right)-k_{i} I_{3}, a_{23}:=k_{i} \ell_{i} I_{3}, a_{24}:=k_{i} I_{3}, a_{43}:=$ $\left(k_{i}-l_{i}\right) \ell_{i} I_{3}$ and $a_{44}:=\left(k_{i}-l_{i}-\ell_{i}\right) I_{3}$. Finally, $B:=\left(B_{l}^{\top}, B_{f}^{\top}\right)^{\top}$ with

$$
B_{l}:=\frac{1}{m_{l}}\left[\begin{array}{ll}
0_{3} & 0_{3} \\
I_{3} & 0_{3} \\
0_{3} & 0_{3} \\
I_{3} & 0_{3}
\end{array}\right] \quad \text { and } \quad B_{f}:=\frac{1}{m_{l} m_{f}}\left[\begin{array}{cc}
0_{3} & 0_{3} \\
-m_{f} I_{3} & m_{l} I_{3} \\
0_{3} & 0_{3} \\
-m_{f} I_{3} & m_{l} I_{3}
\end{array}\right]
$$




\subsection{Robustness of the overall formation}

The following result establishes robustness of the controlled formation to a wide class of disturbances:

Proposition 2. Let Assumption 1 hold. Let the controller of the leader spacecraft be given by (8)-(10) and the controller of the follower spacecraft be given by (11)-(13) with, for each $i \in\{l, f\}, l_{i} \geq 2 k_{i}, k_{i}>2 k_{i}^{\star}$, where

$$
k_{i}^{\star}:= \begin{cases}\ell_{i}+\tilde{\beta}_{i} & \text { if } k_{i}-\ell_{i} \leq k_{i} \ell_{i}^{2} \\ \tilde{\beta}_{i} / \ell_{i}^{2} & \text { otherwise, }\end{cases}
$$

with

$$
\tilde{\beta}_{i}:=\beta_{\dot{v}_{o}} \sqrt{2 \ell_{i}^{2}+1}+\left(1+\frac{m_{f}^{2}}{m_{l}^{2}}\right) \frac{\left(l_{i}^{2}+1\right)}{m_{i}^{2}} .
$$

Given any precision $\delta>0$ and any time window $T>0$, consider any average energy satisfying

$$
E \leq \frac{1}{4} \min _{i \in\{l, f\}}\left\{\ell_{i}^{2}-\frac{1}{2} \sqrt{4 \ell_{i}^{4}+1}+\frac{1}{2}\right\} \delta^{2} \frac{e^{\kappa T}-1}{2 e^{\kappa T}-1}
$$

where

$$
\kappa:=\frac{\min _{i \in\{l, f\}} k_{i}^{\star} / \max _{i \in\{l, f\}}\left\{\frac{k_{i}}{\ell_{i}}\right\}}{\max _{i \in\{l, f\}}\left\{\ell_{i}^{2}+\frac{1}{2} \sqrt{4 \ell_{i}^{4}+1}+\frac{1}{2}\right\}} .
$$

Then, for any $d \in \mathcal{W}_{\gamma}(E, T)$ where $\gamma(s):=s^{2}$, the ball $\mathcal{B}_{\delta}$ is GES for the overall formation summarized by (14).

The proof of the proposition is found in [6].

As already stressed, the results recalled in Section 2 allow to expect more parsimonious solicitation of the actuators than classical ISS-based reasonings. However, such an improvement will only be made practical if the gains of Proposition 2 are tuned in an adequate manner. The rest of the document focuses on this issue.

\section{Optimal controller and observer gain tuning}

Of simplicity we will in the following consider the unperturbed version of (14),

$$
\dot{X}=A\left(\dot{\mathrm{v}}_{o}(t), \theta\right) X,
$$

since $d$ is only characterized by its moving average and therefore difficult to incorporate in the optimization problem we will set forth in the sequel. One possibility to incorporate the disturbance into the optimization problem would be to look at the worst case scenario, which is typical of differential game problems or the inverse optimal gain assignment problem mentioned in the introduction. The worst case excitation due to the disturbances with limited moving average, can for a linear system be found using the 
results of [7]. We however, aim at finding the parameters $\theta$, which are the best compromise between fast state convergence and limited control efforts, while ignoring the effect of the disturbance. If the problem is posed as an optimization problem, a common choice is to use a cost function which quadratically penalizes control and state deviation, i.e. a cost function of the form

$$
J(\theta)=\int_{t_{0}}^{t_{h}} X(\tau)^{\top} Q X(\tau)+\bar{u}(\tau)^{\top} R \bar{u}(\tau) d \tau
$$

with weighting matrices $Q \in \mathbb{R}^{24 \times 24}$ and $R \in \mathbb{R}^{6 \times 6}$ both being symmetric and positive definite, optimization horizon $t_{h}$ and $\bar{u}=\left(\bar{u}_{l}^{\top}, \bar{u}_{f}^{\top}\right)^{\top}$, where

$$
\bar{u}_{l}=m_{l}\left[D\left(\dot{\mathrm{v}}_{o}, \ddot{v}_{o}\right) e_{l}-k_{l}\left(\dot{p}_{0}-\dot{p}_{r}\right)\right]
$$

and

$$
\bar{u}_{f}=m_{f}\left[D\left(\dot{\mathrm{v}}_{o}, \ddot{v}_{o}\right)\left(e_{l}+e_{f}\right)-k_{l}\left(\dot{p}_{0}-\dot{p}_{r}\right)-k_{f}\left(\dot{\rho}_{0}-\dot{\rho}_{r}\right)\right]
$$

are the terms of (8) and (11), respectively, that we want to penalize. It can be noticed, that we have chosen not to penalize the feed-forward terms from the reference and the gravity compensation in (8) and (11). The cost (20), can be written as:

$$
J(\theta)=\int_{t_{0}}^{t_{h}} X^{\top}(\tau)\left(Q+(T+\Xi)^{\top} R(T+\Xi)\right) X(\tau) d \tau
$$

with

$$
T:=m_{f}\left[\begin{array}{cccccccc}
\frac{m_{l}}{m_{f}} k_{l} \ell_{l} I_{3} & \frac{m_{l}}{m_{f}} k_{l} I_{3} & -\frac{m_{l}}{m_{f}} k_{l} \ell_{l} I_{3} & -\frac{m_{l}}{m_{f}} k_{l} I_{3} & 0_{3} & 0_{3} & 0_{3} & 0_{3} \\
k_{l} \ell_{l} I_{3} & k_{l} I_{3} & -k_{l} \ell_{l} I_{3} & -k_{l} I_{3} & k_{f} \ell_{f} I_{3} & k_{f} I_{3} & -k_{f} \ell_{f} I_{3} & -k_{f} I_{3}
\end{array}\right],
$$

and

$$
\Xi:=\left[\begin{array}{cc}
m_{l} D\left(\dot{\mathrm{v}}_{o}, \ddot{\mathrm{v}}_{o}\right) & 0_{3} \\
0_{3} & m_{f} D\left(\dot{\mathrm{v}}_{o}, \ddot{v}_{o}\right)
\end{array}\right]\left[\begin{array}{cccccccc}
I_{3} & 0_{3} & 0_{3} & 0_{3} & 0_{3} & 0_{3} & 0_{3} & 0_{3} \\
I_{3} & 0_{3} & 0_{3} & 0_{3} & I_{3} & 0_{3} & 0_{3} & 0_{3}
\end{array}\right]
$$

We now introduce the state transition matrix $\Phi\left(t, t_{o}\right)$ which is the unique solution of

$$
\frac{\partial}{\partial t} \Phi\left(t, t_{0}\right)=A\left(\dot{\mathrm{v}}_{o}(t), \theta\right) \Phi\left(t, t_{0}\right)
$$

with initial condition

$$
\Phi\left(t_{0}, t_{0}\right)=I_{24}
$$

The solution to (19) is

$$
X(t)=\Phi\left(t, t_{0}\right) X_{0},
$$

and is used to rewrite the cost function into the following form

$$
J(\theta)=X_{0}^{\top} \int_{t_{0}}^{t_{h}} \Phi^{\top}\left(\tau, t_{0}\right)\left(Q+(T+\Xi(\tau))^{\top} R(T+\Xi(\tau))\right) \Phi\left(\tau, t_{0}\right) d \tau X_{0} .
$$


We will avoid dependency of the cost function on initial conditions, and will assume that $X_{0}$ are randomized variables with zero mean, and covariance matrix equal to identity, that is $\mathrm{E}\left\{X_{0} X_{0}^{\top}\right\}=I_{24}$. By defining

$$
P\left(t_{h}, t_{0}\right):=\int_{t_{0}}^{t_{h}} \Phi^{\top}\left(\tau, t_{0}\right)\left(Q+(T+\Xi(\tau))^{\top} R(T+\Xi(\tau))\right) \Phi\left(\tau, t_{0}\right) d \tau
$$

and using the fact that

$$
\begin{aligned}
\mathrm{E}\left\{X_{0}^{\top} P\left(t_{h}, t_{0}\right) X_{0}\right\} & =\mathrm{E}\left\{\operatorname{trace} X_{0}^{\top} P\left(t_{h}, t_{0}\right) X_{0}\right\} \\
& =\mathrm{E}\left\{\operatorname{trace} X_{0} X_{0}^{\top} P\left(t_{h}, t_{0}\right)\right\} \\
& =\operatorname{trace} \mathrm{E}\left\{X_{0} X_{0}^{\top}\right\} P\left(t_{h}, t_{0}\right) \\
& =\operatorname{trace} P\left(t_{h}, t_{0}\right),
\end{aligned}
$$

our optimization problem can be formulated as

$$
\min _{\theta} \mathrm{E}\{J(\theta)\}=\min _{\theta} \operatorname{trace} P\left(t_{h}, t_{0}\right)
$$

subject to

$$
\frac{\partial}{\partial t} \Phi\left(t, t_{0}\right)=A\left(\dot{\mathrm{v}}_{o}(t), \theta\right) \Phi\left(t, t_{0}\right)
$$

with $\Phi\left(t_{0}, t_{0}\right)=I_{24}$,

$$
\left.\frac{\partial}{\partial t} P\left(t, t_{0}\right)=\Phi^{\top}\left(t, t_{0}\right)\left(Q+(T+\Xi(t))^{\top}\right) R(T+\Xi(t))\right) \Phi\left(t, t_{0}\right),
$$

with $P\left(t_{0}, t_{0}\right)=\left(Q+\left(T+\Xi\left(t_{0}\right)\right)^{\top} R\left(T+\Xi\left(t_{0}\right)\right)\right)$, for each $i \in\{l, f\}$,

$$
\begin{aligned}
& l_{i} \geq 2 k_{i} \\
& k_{i}>2 k_{i}^{\star}
\end{aligned}
$$

where

$$
k_{i}^{\star}:= \begin{cases}\ell_{i}+\tilde{\beta}_{i} & \text { if } k_{i}-\ell_{i} \leq k_{i} \ell_{i}^{2} \\ \tilde{\beta}_{i} / \ell_{i}^{2} & \text { otherwise }\end{cases}
$$

with

$$
\tilde{\beta}_{i}:=\beta_{\dot{v}_{o}} \sqrt{2 \ell_{i}^{2}+1}+\left(1+\frac{m_{f}^{2}}{m_{l}^{2}}\right) \frac{\left(l_{i}^{2}+1\right)}{m_{i}^{2}}
$$

and

$$
\begin{aligned}
k_{l}, \ell_{l}, k_{f}, \ell_{f} & \in[0,2] \\
l_{l} & \in[0,10] \\
l_{f} & \in[0,15] .
\end{aligned}
$$

The constraints (38)-(40) are introduced to more efficiently solve the problem. The problem was solved using the interior point algorithm of the function fmincon in MATLAB. 
The true anomaly, $v_{o}$, of the reference frame can be obtained by numerical integration of the equation

$$
\dot{\mathrm{v}}_{o}(t)=\frac{\sqrt{\mu}\left(1+e_{o} \cos v_{o}(t)\right)^{2}}{\left(\frac{1}{2}\left(r_{a}+r_{p}\right)\left(1-e_{o}^{2}\right)\right)^{3 / 2}},
$$

where radius of perigee and apogee is chosen as $r_{p}=10^{7} \mathrm{~m}$ and $r_{a}=3 \times 10^{7} \mathrm{~m}$, respectively. The true anomaly rate and rate of change are calculated using (41) and

$$
\ddot{\mathrm{v}}_{o}(t)=\frac{-2 \mu e_{o}\left(1+e_{o} \cos \mathrm{v}_{o}(t)\right)^{3} \sin \mathrm{v}_{o}(t)}{\left(\frac{1}{2}\left(r_{p}+r_{a}\right)\left(1-e_{o}^{2}\right)\right)^{3}} .
$$

The eccentricity can be calculated from $r_{a}$ and $r_{p}$ to be $e_{o}=0.5$, and we see from (42), that the constant $\beta_{\ddot{v}_{o}}$ in Assumption 1 can be chosen as $\beta_{\ddot{v}_{o}}=4 \times 10^{-7}$. The constant $\beta_{\dot{v}_{o}}$ in Assumption 1 can be chosen as $\beta_{\dot{v}_{o}}=8 \times 10^{-4}$ as seen from (41). We have assumed that the reference frame is initially at perigee, that is $v_{o}\left(t_{0}\right)=0$ and $\dot{v}_{o}\left(t_{0}\right)=v_{p} / r_{p}$, where

$$
v_{p}=\sqrt{2 \mu\left(\frac{1}{r_{p}}-\frac{1}{\left(r_{p}+r_{a}\right)}\right)} .
$$

The horizon of the problem was chosen as $t_{h}=30$, and the optimization problem was solved using $R=I_{24}$ and $Q$ with ones along the diagonal except that $Q(2,2)=Q(3,3)=$ $Q(14,14)=Q(15,15)=20$. It can be argued that choosing the elements of $R$ and $Q$ is as hard as finding the parameters $\theta$ in the original problem. However, with the common choice of diagonal $Q$ and $R$, choosing $Q$ and $R$ is more intuitive in the authors opinion. The optimization problem, was solved with all combinations of initial conditions $\theta_{i} \in\{0,1,2\}$, and the results are summarized in Table 1 . The reason for solving

\begin{tabular}{c|c|c|c|c|c|c}
\hline Parameter & $k_{l}$ & $\ell_{l}$ & $l_{l}$ & $k_{f}$ & $\ell_{f}$ & $l_{f}$ \\
\hline Best value & 0.3382 & 0.2658 & 2.0048 & 0.3738 & 0.3302 & 1.7644 \\
Mean & 0.3280 & 0.2809 & 1.6541 & 0.3855 & 0.3154 & 1.8660 \\
\hline
\end{tabular}

Table 1. Optimization result of 729 iterations, covering all combinations of initial conditions $\theta_{i} \in\{0,1,2\}$. By Best value, we mean the parameters which gives the lowest cost function value.

the optimization problem with several different initial values, is to find, if not a global minimum, then at least a good local minimum. Looking at mean values in Table 1, we see that the parameters $l_{l}$ and $l_{f}$ are most affected by different initial guesses for the parameters.

The parameters giving the lowest cost function were then used as initial guess, and the problem was solved with different values for the horizon $t_{h}$. The results in Table 2 suggests that the found parameters represent a good local minimum.

\subsection{Simulations}

We will now provide simulations of the spacecraft formation, using the gains achieved from the optimization procedure in the previous section. For simplicity, we choose 


\begin{tabular}{c|c|c|c|c|c|c}
\hline Horizon & $k_{l}$ & $\ell_{l}$ & $l_{l}$ & $k_{f}$ & $\ell_{f}$ & $l_{f}$ \\
\hline 20 & 0.3195 & 0.2743 & 1.5802 & 0.3829 & 0.3200 & 1.8948 \\
50 & 0.3382 & 0.2658 & 2.0048 & 0.3738 & 0.3302 & 1.7644 \\
100 & 0.3382 & 0.2658 & 2.0048 & 0.3738 & 0.3302 & 1.7644 \\
300 & 0.3415 & 0.3089 & 1.9524 & 0.3631 & 0.3245 & 1.7608 \\
1000 & 0.3382 & 0.2658 & 2.0048 & 0.3738 & 0.3302 & 1.7644 \\
\hline
\end{tabular}

Table 2. Optimization result with different values of the horizon length $t_{h}$.

the desired trajectory of the leader spacecraft to coincide with the reference orbit, i.e. $p_{d}(\cdot) \equiv(0,0,0)^{\top}$. The reference orbit is generated by numerical integration of

$$
\ddot{r}_{o}=-\frac{\mu}{\left|r_{o}\right|^{3}} r_{o}
$$

with $r_{o}(0)=\left(r_{p}, 0,0\right)$ and $\dot{r}_{o}(0)=\left(0, v_{p}, 0\right)$, with $v_{p}$ as in (43). Initial values of the leader spacecraft are $p(0)=(2,-2,3)^{\top}$ and $\dot{p}(0)=(0.4,-0.8,-0.2)^{\top}$. The initial values of the observer are chosen as $\hat{p}(0)=(0,0,0)^{\top}$ and $a_{l}(0)=(0,0,0)^{\top}$.

The reference trajectory of the follower spacecraft are chosen as the solutions of a special case of the Clohessy-Wiltshire equations, cf. [3]. We use

$$
\rho_{d}(t)=\left[\begin{array}{c}
10 \cos v_{o}(t) \\
-20 \sin v_{o}(t) \\
0
\end{array}\right]
$$

This choice imposes that the two spacecraft evolve in the same orbital plane, and that the follower spacecraft makes a full rotation about the leader spacecraft at each orbit around the Earth. The initial values of the follower spacecraft are $\rho(0)=(9,-1,2)^{\top}$ and $\dot{\rho}(0)=(-0.3,0.2,0.6)^{\top}$. The initial parameters of the observer are chosen to be $\hat{\rho}(0)=\rho_{d}(0)=(10,0,0)^{\top}$ and $a_{f}(0)=(0,0,0)^{\top}$. We use $m_{f}=m_{l}=25 \mathrm{~kg}$ both in the model and the control structure.

With $\theta$ as the best value in Table 1 , we find from (18) that $\kappa=0.0901$. Over a 10 second interval (i.e. $\mathrm{T}=10$ ), the average excitation must satisfy $E(T, \delta) \leq 0.0061 \delta^{2}$, according to (17). We consider two types of disturbances acting on the spacecraft: "impacts" and continuous disturbances. The "impacts" have random amplitude, but with maximum of $1.5 \mathrm{~N}$ in each direction of the Cartesian frame. For simplicity, we assume that at most one impact can occur over each 10 second interval, and we assume that the duration of each impact is at most $0.1 \mathrm{~s}$. The continuous disturbances are taken as sinusoids, also acting in each direction of the Cartesian frame, and are chosen to be $(0.1 \sin 0.01 t, 0.25 \sin 0.03 t, 0.3 \sin 0.04 t)^{\top}$ for both spacecraft. Notice from (7) that the relative dynamics are influenced by disturbances acting on the leader and follower spacecraft, so the effect of the continuous part of the disturbance on the relative dynamics is zero. It can easily be shown that the disturbances satisfy the following:

$$
\int_{t}^{t+10}|d(\tau)|^{2} d \tau \leq 1.42, \quad \forall t \geq 0 .
$$




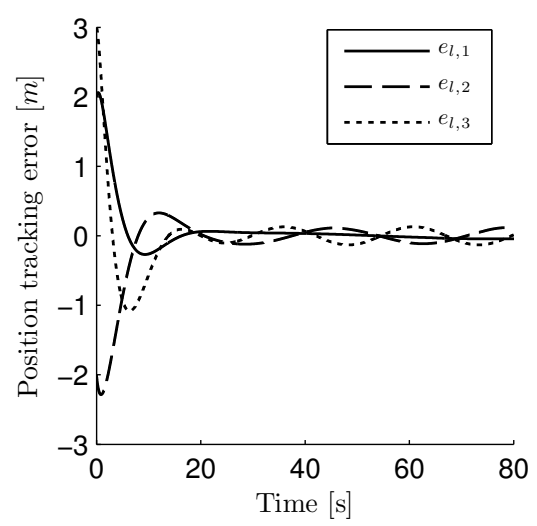

(a) Position tracking error

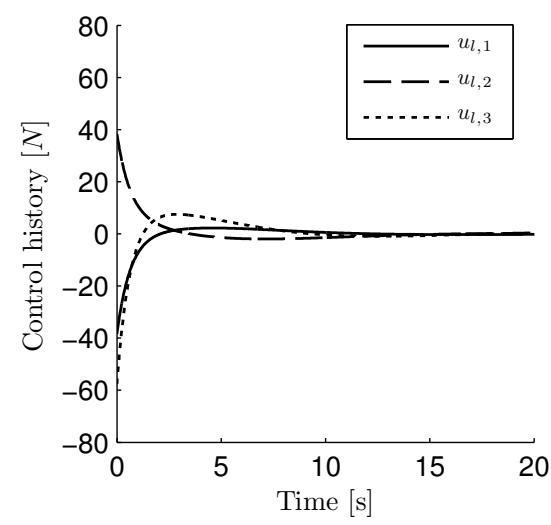

(c) Control history

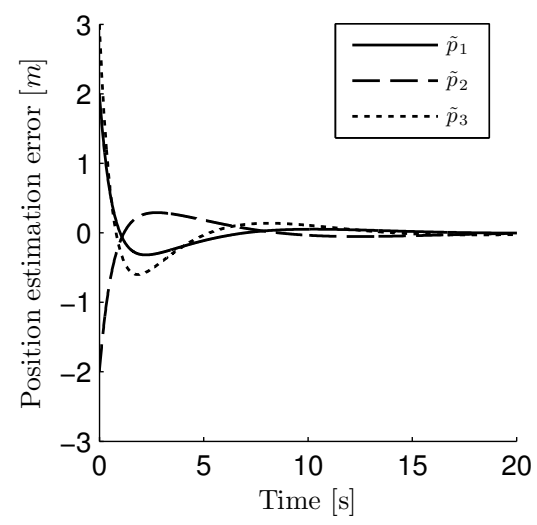

(b) Position estimation error

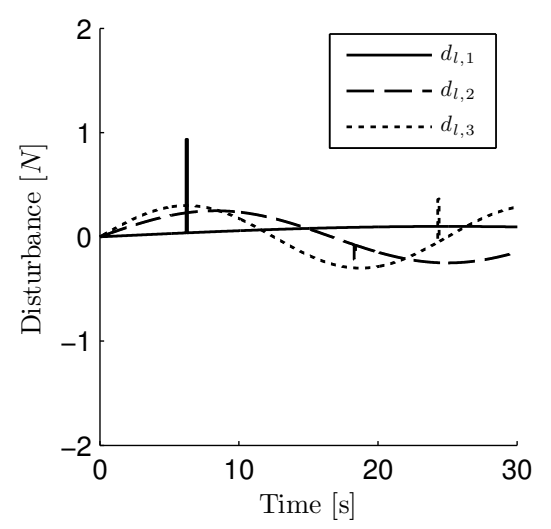

(d) Disturbance history

Fig. 1. Leader spacecraft.

Figure 1(a), 1(b) and 1(c) show the position tracking error, position estimation error and control history of the leader spacecraft, whereas Figure 2(a), 2(b) and 2(c) are the equivalent figures for the follower spacecraft. Figure 1(d) and 2(d) show the effect of $d_{l}$ and $d_{f}-d_{l}$ acting on the formation. Notice in Figure 2(d) that the effect of the continuous part of the disturbance is canceled out (since we consider relative dynamics and both spacecraft are influenced by the same continuous disturbance), whereas the effect of the impacts has increased compared to the effect of the impacts on the leader spacecraft. Since $E=1.42$, and should satisfy $E \leq 0.0061 \delta^{2}$, this gives a very large $\delta$. As can be seen from Figure 1(a), 1(b), 2(a), 2(b) the actual precision reached, is much better than the theoretical expectations. The reason for this is that the constraints on the control gains are based on Lyapunov analysis, which in general yields very conservative results, and also conservative estimates of the disturbances the control system is able to 


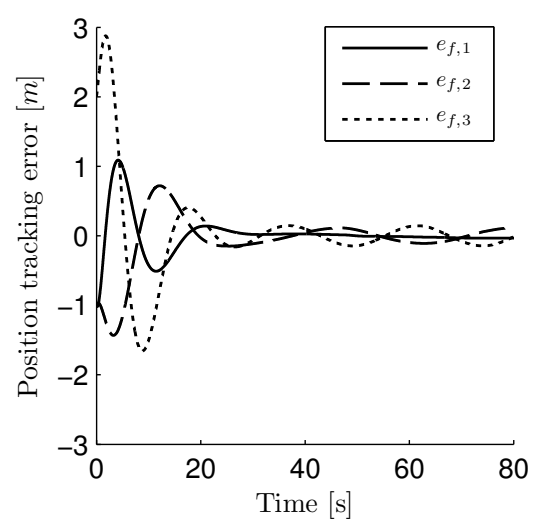

(a) Position tracking error

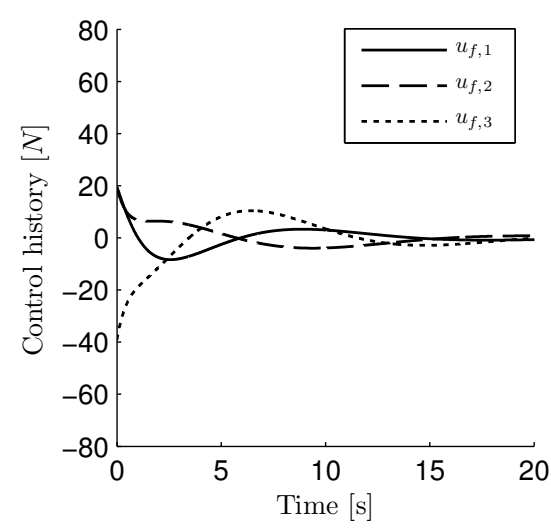

(c) Control history

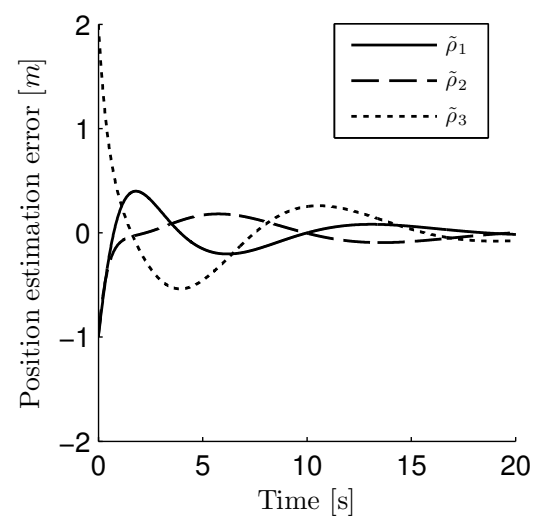

(b) Position estimation error

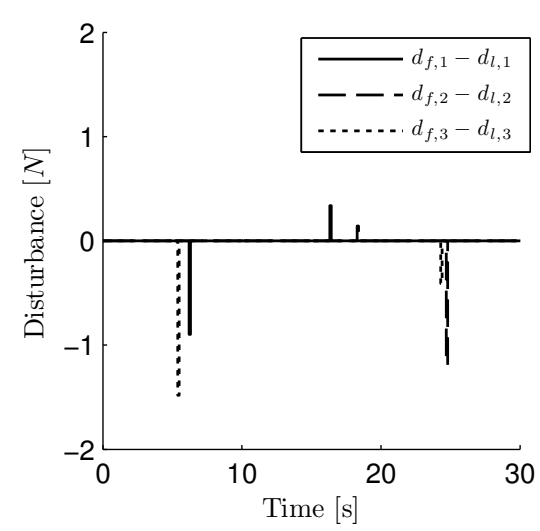

(d) Disturbance history

Fig. 2. Follower spacecraft.

handle. However, we stress that the constraints on the gains based on moving average of the disturbance are much more relaxed than those obtained through a classical ISS approach, i.e. relying on the disturbance magnitude. Figure 1(c) and Figure 2(c), shows that the conservative estimates results in large transients in the actuation, but by finding the optimal parameters, the control efforts are heavily reduced compared to [6], while the tracking errors are kept at a reasonable level.

\section{References}

1. Angeli, D., Nešić, D.: Power characterizations of input-to-state stability and integral inputto-state stability. IEEE Transactions on Automatic Control 48, 1298-1303 (2001)

2. Brockett, R.W.: Finite Dimensional Linear Systems. Wiley, New York (1970)

3. Clohessy, W.H., Wiltshire, R.S.: Terminal guidance system for satellite rendezvous. Journal of Aerospace Sciences 27, 9 (1960) 
4. Doyle, J.: Robust and optimal control. In: Proc. of the 35th Conference on Decision and Control (1996)

5. Freeman, R.A., Kokotovic̀, P.V.: Robust nonlinear control design: state-space and Lyapunov techniques. Birkhuser (1996)

6. Grøtli, E.I., Chaillet, A., Panteley, E., Gravdahl, J.T.: Robustness of ISS systems to inputs with limited moving average, with application to spacecraft formations. In: Proc. of the 7th International Conference on Informatics in Control, Automation and Robotics (2010)

7. Houska, B., Diehl, M.: Robust nonlinear optimal control of dynamic systems with affine uncertainties. In: Proc. of the 48th IEEE Conference on Decision and Control and 28th Chinese Control Conference (2009)

8. Kazantizis, N., Kravaris, C., Tseronis, C., Wright, R.A.: Optimal controller tuning for nonlinear processes. Automatica 41, 79-86 (2005)

9. Kristiansen, R., Loría, A., Chaillet, A., Nicklasson, P.J.: Adaptive output feedback control of spacecraft relative translation. In: Proc. of the 45th Conference on Decision \& Control. pp. 6010-6015 (2006)

10. Krstić, M., Li, Z.H.: Inverse optimal design of input-to-state stabilizing nonlinear controllers. IEEE Transactions on Automatic Control 43, 336-350 (1998)

11. Ploen, S.R., Scharf, D.P., Hadaegh, F.Y., Acikmese, A.B.: Dynamics of earth orbiting formations. In: Proc. of AIAA Guidance, Navigation and Control Conference (2004)

12. Praly, L., Wang, Y.: Stabilization in spite of matched unmodeled dynamics and an equivalent definition of input-to-state stability. Mathematics of Control, Signals, and Systems 9, 1-33 (1996)

13. van der Schaft, A.J.: $\mathcal{L}_{2}$-gain analysis of nonlinear systems and nonlinear state feedback $\mathcal{H}_{\infty}$ control. IEEE Transactions on Automatic Control 37, 770-784 (1992)

14. Serrani, A.: Robust coordinated control of satellite formations subject to gravity perturbations. In: Proc. of the American Control Conference. pp. 302-307 (2003)

15. Sontag, E.D.: Smooth stabilization implies coprime factorization. IEEE Transactions on Automatic Control 34, 435-443 (1989)

16. Sontag, E.D.: A 'universal' construction of Artstein's theorem on nonlinear stabilization. Systems \& Control Letters 13, 117-123 (1989)

17. Sontag, E.D., Wang, Y.: On characterizations of the input-to-state stability property. Systems \& Control Letters 24, 351-359 (1995)

18. Tadmor, G.: Worst-case design in the time domain: The maximum principle and the standard $\mathcal{H}_{\infty}$ problem. Math. Control Signals Systems 3, 1989 (1989)

19. Zames, G.: Feedback and optimal sensitivity: model reference transformations, multiplicative seminorms, and approximate inverses. IEEE Transactions on Automatic Control 26, 301-320 (1981) 